TRANSACTIONS OF THE

AMERICAN MATHEMATICAL SOCIETY

Volume 296, Number 2, August 1986

\title{
WORD MAPS, ISOTOPY AND ENTROPY
}

\author{
DAVID FRIED ${ }^{1}$
}

\begin{abstract}
We find diffeomorphisms of low entropy in each isotopy class on $S^{3} \times S^{3}$. These arise as word maps, a nonabelian analogue of toral automorphisms. Hyperbolic examples of equal entropy are also found. The group $\pi_{0} \operatorname{Diff}\left(S^{3} \times S^{3}\right)$ is computed.
\end{abstract}

The rule $x_{n+1}=x_{n}+x_{n-1}$ that generates the Fibonacci sequence can be applied to any initial pair of integers $x_{1}, x_{2}$ : Fibonacci used 1, 1, Lucas used 1, 3, etc. One can use the corresponding multiplicative rule $x_{n+1}=x_{n} x_{n-1}$ for any group $H$ and any initial pair of group elements. This defines a sort of discrete delay equation, or a "second order" transformation on $H$, that can be viewed as a "firstorder" transformation $\sigma$ on pairs of elements: $\sigma(x, y)=(y, y x), \sigma: H \times H \hookleftarrow$. The Fibonacci and Lucas series are two orbits of $\sigma$ for $H=\mathbf{Z}$.

This map $\sigma$ is an instance of what we call a word map on two letters, since it assigns to the letters $x, y$ a new pair of words in $x, y$ and their inverses. Note $\sigma$ is invertible and $\sigma^{-1}$ is also a word map, $\sigma^{-1}(x, y)=\left(x^{-1} y, x\right)$. Such invertible word maps on two letters are determined by automorphisms of the free group $F_{2}=F\left(x_{1}, x_{2}\right)$ on two generators. For example, $\sigma$ corresponds to the automorphism sending $x_{1}$ to $x_{2}$ and $x_{2}$ to $x_{2} x_{1}$. Similar remarks hold for word maps on $n$ letters, $n \geq 1$.

The case $H=S^{1}$ is well known, since here an invertible word map on $n$ letters is just an automorphism of the torus $T^{n}$ of dimension $n$. These toral automorphisms are among the best-understood dynamical systems. Our interest is in the case $H$ a compact connected Lie group and comparing its invertible word maps with toral automorphisms.

Suppose, for example, that $H$ is the group $S O(d)$ of rotations of Euclidean space of dimension $d$. Then $\sigma$ defines a transformation $\sigma_{d}$ for each $d$. The case $d=2$ gives the toral automorphism $\left(\begin{array}{ll}0 & 1 \\ 1 & 1\end{array}\right)$ with topological entropy $h\left(\sigma_{2}\right)=\log \rho, \rho$ the Golden Ratio $(1+\sqrt{5}) / 2$. In $\S 1$, we will show that, surprisingly, $h\left(\sigma_{3}\right)=h\left(\sigma_{2}\right)$. In this sense, the Fibonacci transformation on $S O(3)$ is no more complicated than that for dimension 2. One has $h\left(\sigma_{4}\right)=2 h\left(\sigma_{2}\right), h\left(\sigma_{d}\right) \geq[d / 2] h\left(\sigma_{2}\right)$. The same holds true for any invertible word map. We do not know $h\left(\sigma_{d}\right)$ for $d \geq 5$.

Our main interest is in invertible word maps on $H=S^{3}$ on two letters. Besides computing their entropy we show in $\S 2$ that they furnish representatives for all isotopy classes of the 6-manifold $M=S^{3} \times S^{3}$. This determines the group structure of $\pi=\pi_{0}$ Diff $M$, the group of isotopy classes of diffeomorphisms of $M$. Previously one knew a certain three-step filtration of $\pi$ without knowing what the extensions

Received by the editors August 26, 1985.

1980 Mathematics Subject Classification (1985 Revision) Primary 58F15, 57R52, 28 D20.

${ }^{1}$ Partially supported by the Sloan Foundation and the National Science Foundation. 
were: indeed, Kreck gave such a description for a large class of smooth manifolds $[\mathbf{K r}]$.

This new information on $\pi$ has a pleasant dynamical consequence. The natural homomorphism Diff $M \rightarrow G l_{2} \mathbf{Z}$ that assigns to diffeomorphism its action on $H_{3}(M ; \mathbf{Z}) \cong \mathbf{Z}^{2}$ is onto, just like the homomorphism Diff $T^{2} \rightarrow G l_{2} \mathbf{Z}$. But whereas the linear action of $G l_{2} \mathbf{Z}$ on $T^{2}$ splits the latter map, there is no splitting of the former. In fact we show there is not even a splitting modulo isotopy; i.e., the extension $\pi \rightarrow G l_{2} \mathbf{Z}$ does not split. This depends critically on the existence of exotic 7-spheres.

Given a diffeomorphism $f$ of a compact manifold $X$, we let $s(f)$ be the spectral radius of the homology map $f_{*}: H_{*}(X ; \mathbf{R}) \hookleftarrow$. Shub's entropy conjecture $[\mathbf{S}]$ is that $h(f) \geq \log s(f)$. On a simply connected manifold, $\log s(f)$ is the only topological constant available that might so bound entropy. This conjecture is not known for $X=M$, but our results show that each isotopy class on $M$ has a representative $f$ with $h(f)=\log s(f)$. So, modulo this difficult conjecture, such $f$ 's should be representatives of greatest "efficiency". ${ }^{2}$

We go further (in $\S 3$ ) and show that $f$ can be chosen structurally stable. We do this by an isentropic deformation of a word map, i.e., one that does not change entropy. This $f$ can even be chosen fitted, which is compatible with conjectures of Shub that the lowest entropy occurs for fitted diffeomorphisms, which are hyperbolic transformations built using handlebodies $[\mathbf{S}]$. Some counterexamples for $X$ not simply connected are known, e.g., for $X=T^{4}$; these also use word maps [F2]. It is conceivable that these give counterexamples on $M \times M=\left(S^{3}\right)^{4}$.

Thus, in passing from $T^{2}$ to $M$, the behavior of entropy in an isotopy class seems to persist. But we do not even know that word maps are points of lower semicontinuity for entropy on Diff $M$, let alone that they are minima on their components. It would be interesting to show they are local minima.

In the course of our computations, we find a surprising deformation of the action of $P G l_{2} \mathbf{Z}=\Gamma$ on $T^{2} / \pm 1$ induced from the linear action of $G l_{2} \mathbf{Z}$ on $T^{2}$. The deformation passes through analytic actions of $\Gamma$ on $S^{2}$ that preserve area, and it ends with a finite group of orthogonal motions of $S^{2}$. We do not know whether the intermediate actions are ergodic.

We thank Dan Asimov and Bill Goldman for their interesting observations.

1. Word maps. Let $F_{n}$ be the free group on generators $x_{1}, \ldots, x_{n}$, and let $\Phi_{n}$ be the automorphism group of $F_{n}$. Given any group $H$, there is a natural bijection of $H^{n}$ with the collection $\operatorname{Hom}\left(F_{n}, H\right)$ of homomorphisms from $F_{n}$ to $H$ given by evaluating the homomorphism on the generators. This gives an action of $\Phi_{n}$ on $H^{n}$. If $\alpha \in \Phi_{n}$, then we denote the corresponding bijection of $H^{n}$ by $\alpha_{H}$. We call $\alpha_{H}$ a word map on $n$ letters since it assigns to an $n$-tuple in $H$ an $n$-tuple of words in the entries.

For example, suppose $n=2, \alpha\left(x_{1}\right)=x_{2}$ and $\alpha\left(x_{2}\right)=x_{2} x_{1}$. Then $\alpha_{H}\left(h_{1}, h_{2}\right)=$ $\left(h_{2}, h_{2} h_{1}\right)$ for $h_{1}, h_{2} \in H$, so $\alpha_{H}=\sigma$ is the Fibonacci map discussed above.

When $H=\mathbf{R}$ or $\mathbf{C}$, the $\alpha_{H}$ are linear maps. Indeed, when $H$ is abelian, $\Phi_{n}$ acts by group automorphisms and the action factors through $G l_{n} \mathbf{Z}$, the automorphism group of the abelianization of $F_{n}$.

\footnotetext{
${ }^{2}$ Yomdin has recently proven the conjecture for $C^{\infty} f$.
} 
For general $H, H$ acts on $H^{n}$ by conjugation:

$$
h \cdot\left(h_{1}, \ldots, h_{n}\right)=\left(h h_{1} h^{-1}, \ldots, h h_{n} h^{-1}\right) .
$$

Let $C_{n}=C_{n}(H)$ be the orbit space. Then the actions of $\Phi_{n}$ and $H$ on $H^{n}$ commute, so $\Phi_{n}$ acts on $C_{n}$. In this action, inner automorphisms act trivially, and so there is an induced action of the outer automorphism group Out $F_{n}\left(=\Phi_{n} / F_{n}\right.$ for $\left.n \geq 2\right)$ on $C_{n}$. We denote by $\bar{\alpha}_{H}$ the map on $C_{n}$ induced by $\alpha_{H}$.

Now we fix $n=2$. We need some results due to Nielsen [N, MKS]. First, each $\alpha \in \Phi_{2}$ "preserves" the commutator of $x_{1}^{-1} x_{2}^{-1} x_{1} x_{2}=c\left(x_{1}, x_{2}\right)$; i.e., $\alpha\left(c\left(x_{1}, x_{2}\right)\right)=$ $g c\left(x_{1}, x_{2}\right)^{\varepsilon} g^{-1}$, for some $\varepsilon= \pm 1, g \in F_{2}$. This implies that the commutator map $c: H^{2} \rightarrow H, c(x, y)=x^{-1} y^{-1} x y$, induces a map $\bar{c}: C_{2} \rightarrow C_{1}$ that is equivariant tor the actions of Out $F_{i}, i=1,2$, under the homomorphism $\varepsilon: F_{2} \rightarrow$ Out $F_{1}$. Second, the natural map Out $F_{2} \rightarrow G l_{2} \mathbf{Z}$ is an isomorphism. So $\operatorname{ker}(\varepsilon)=S l_{2} \mathbf{Z}$ acts on $C_{2}$, and $\bar{c}$ is a $S l_{2} \mathbf{Z}$-invariant function. Note $\bar{c}$ is constant iff $H$ is abelian.

Now we fix a compact connected Lie group $H$. Let $T$ be a maximal torus and $W=N(T) / T$ the Weyl group, where $N(T)$ is the normalizer of $T$ in $H$. For the obvious action of $W$ on $T$, it is well known that the map $T / W \rightarrow C_{1}$ is a homeomorphism, where $T / W$ is the orbit space of $T$ under the finite group $W$. This identifies the image of $\bar{c}$ with the orbifold $T / W$ of dimension $r=\operatorname{dim} T$.

We trivialize the tangent space of $H^{2}$ by left translation and fix an ad-invariant inner product on the Lie algebra $\nLeftarrow$ of $H$. This gives $H, H^{2}$ bi-invariant Riemannian metrics. Then the permutation $\alpha\left(x_{1}\right)=x_{2}, \alpha\left(x_{2}\right)=x_{1}$ determines an isometry $\alpha_{H}$ of $H^{2}$, and the shear $\beta\left(x_{1}\right)=x_{1} x_{2}, \beta\left(x_{1}\right)=x_{2}$ has differential $T \beta_{H}=\left(\begin{array}{cc}\Delta & 0 \\ I & I\end{array}\right)$ at $\left(h_{1}, h_{2}\right)$, where $\Delta=\operatorname{ad} h_{2} \in O(\not H)$. Taking $\gamma \in \Phi_{2}$ to be any positive word in $\alpha, \beta$, we find

$$
T \gamma_{H}=\left(\begin{array}{ll}
A_{11} & A_{12} \\
A_{21} & A_{22}
\end{array}\right),
$$

where the operator norms of $A_{i j}$ satisfy $\left\|A_{i j}\right\| \leq a_{i j}$, where the $a_{i j}$ are the entries of the image $\gamma_{\mathbf{R}}$ of $\gamma$ in $G l_{2} \mathbf{Z} \subset G l_{2} \mathbf{R}$. This implies that $\left\|T \gamma_{H}\right\| \leq\left\|\gamma_{\mathbf{R}}\right\|$ at all points of $H^{2}$.

Because the action of $H$ on $H^{2}$ is isometric, there is an induced Riemannian metric on the dense open set $O$ of points in $C_{2}$ with least isotropy. This gives a Riemannian metric on a regular level of $\bar{c}_{2}$ in 0 on which $\left\|T \bar{\gamma}_{H}\right\| \leq\left\|\gamma_{\mathbf{R}}\right\|$

But any $\alpha$ in $S l_{2} \mathbf{Z}$ of infinite order is conjugate to $\pm \gamma, \gamma$ of the above form. We compute in $P S l_{2} \mathbf{Z}=S l_{2} \mathbf{Z} / \pm 1 \cong\left\langle a, b \mid a^{2}=b^{3}=1\right\rangle$, where $a= \pm\left(\begin{array}{cc}0 & 1 \\ -1 & 0\end{array}\right), b=$ $\pm\left(\begin{array}{cc}0 & 1 \\ -1 & -1\end{array}\right)$. Then $a b= \pm\left(\begin{array}{ll}1 & 1 \\ 0 & 1\end{array}\right)$ and $a b^{-1}= \pm\left(\begin{array}{ll}1 & 0 \\ 1 & 1\end{array}\right)$. One can conjugate any element of infinite order to $\prod_{i}(a b)^{c_{i}}\left(a b^{-1}\right)^{d_{i}}$. Now write $a b^{-1}= \pm \beta_{\mathbf{Z}}$ and $a b= \pm \alpha_{\mathbf{Z}} \beta_{\mathbf{Z}} \alpha_{\mathbf{Z}}$.

It follows that for any $\alpha,\left\|T \alpha_{H}^{n}\right\|$ is of the order $\left\|\alpha_{\mathbf{R}}^{n}\right\|$, for all $n>0$, hence of the order $r_{\alpha}^{n}$, where $r_{\alpha}$ is the spectral radius of $\alpha_{\mathbf{R}}$.

We specialize to the case $H=S^{3}$, the group of unit quaternions. In this case we can identify $C_{2}$ with a compact region $P$ in $\mathbf{R}^{3}$ via the real part of the graph of the group law

$$
\tau[x, y]=(\operatorname{Re} x, \operatorname{Re} y, \operatorname{Re} x y),
$$

where $[x, y]$ denotes the conjugacy class in $C_{2}$ of the pair $(x, y) \in S^{3} \times S^{3}$. Clearly $\tau$ is well defined because the real part of a quaternion is invariant under conjugation. 
One can check that $\tau$ is $1-1$ by conjugating $(x, y)$ to normal form with $x \in \mathbf{C}$ and $y \in \mathbf{C}+\mathbf{R} j$.

We identify $C_{1}$ with $[0,1]$ by the map $N[x]=\|1-x\|^{2} / 4$. Then $\bar{c}: C_{2} \rightarrow C_{1}$ can be factored through the cubic polynomial

$$
p(r, s, t)=1-\left(r^{2}+s^{2}+t^{2}\right)+2 r s t
$$

i.e., $p \tau[x, y]=N \bar{c}[x, y]$. Again this is easily checked in normal form. One sees that $p$ has critical points $(0,0,0)$ and $\left(\varepsilon_{1}, \varepsilon_{2}, \varepsilon_{3}\right)$, where $\left|\varepsilon_{i}\right|=1, \varepsilon_{1}, \varepsilon_{2}, \varepsilon_{3}=1$. It is clear that $P$ lies in $[-1,1]^{3} \cap p^{-1}[0,1]$, and one checks these are equal. This was noted independently by Dan Asimov, who observed that $P$ is the region spanned by the three possible pairs of correlations of three independent random variables. He coined the term "tetrahedral pillow" for $P$. This captures the fact that $P$ is obtained from a tetrahedron inscribed in $[-1,1]^{3}$ by rounding the edges so that only the four vertices are singularities of $\partial P$. Note that $P$ meets each side of this cube in a diagonal segment.

We denote the level set $p^{-1}(v) \cap P$ by $L_{v}$. For $v=0, L_{0}=\partial P \cong S^{1} \times S^{1} / \pm 1$ under $\tau, S^{1} \subset S^{3}$ any one parameter subgroup. For $0<v<1, L_{t}$ is an analytic $S^{2}$. For $v=1, L_{1}$ is the origin.

The action of $G l_{2} \mathbf{Z}$ preserves these levels. The action on $\partial P=L_{0}$ is the usual action on $T^{2} / \pm 1$ (note the vertex singularities in $\partial P$ correspond to the fixed points of the involution of $T^{2}$ ).

The action of $G l_{2} \mathbf{Z}$ on $P$ extends to a polynomial action on $\mathbf{R}^{3}$ that preserves volume. This can be checked on generators using the normal form: e.g., for the shear $\beta$,

$$
\bar{\beta}_{H}(r, s, t)=(t, s, 2 s t-r) .
$$

It follows that each level $L_{v}, 0<v<1$, inherits an analytic area form invariant by $\Gamma$.

Now we use S. Katok's theorem that the entropy of a diffeomorphism $f$ of a compact manifold is bounded by $\log s\left(f^{\#}\right)$, where $f^{\#}$ is the action of $f$ on differential forms and $s$ denotes spectral radius $[\mathbf{K}]$. Using the invariant area, we see that the action $f_{2}^{\#}$ of $f=\bar{\alpha}_{S^{3}} \mid L_{v}, 0<v<1$, on 2-forms has spectral radius zero. Because the same holds trivially for 0 -forms, $s\left(f^{\#}\right)=s\left(f_{1}^{\#}\right) \leq \log r_{\alpha}$, by our estimates on $\left\|T \alpha_{S^{3}}^{n}\right\|, n>0$. Altogether $h(f) \leq \log r_{\alpha}$.

For $v=0, h\left(\bar{\alpha}_{S^{3}} \mid S_{0}\right)=h\left(\bar{\alpha}_{S^{1}}\right)=\log r_{\alpha}$. Thus by [B, Corollary 18], $h\left(\bar{\alpha}_{S^{3}}\right)=$ $\sup _{v} h\left(\bar{\alpha}_{S^{3}} \mid L_{v}\right)=\log r_{\alpha}$. Since $\bar{\alpha}_{S^{3}}$ is the factor of $\alpha_{S^{3}}$ by the action of the compact group $H$ and the entropy of $\alpha_{S^{3}}$ on each $H$-orbit is clearly zero, [B, Theorem 17] implies $h\left(\alpha_{S^{3}}\right)=h\left(\bar{\alpha}_{S^{3}}\right)=\log r_{\alpha}$.

It follows that for all $\alpha \in \Phi_{2}$ (even with $\varepsilon=-1$ ) $h\left(\alpha_{S^{3}}\right)=\log r_{\alpha}$, since both sides double when $\alpha$ is replaced by $\alpha^{2}$. Note that $\Phi_{1}$ acts trivially on $C_{1}$, so the action of $G l_{2} \mathbf{Z}$ on $C_{2}$ leaves $\bar{c}$ invariant.

For $f=\alpha_{S^{3}}$, the action of $f_{*}$ on $H^{3}\left(S^{3} \times S^{3} ; \mathbf{Z}\right) \cong \mathbf{Z}^{2}$ is just $\alpha_{\mathbf{Z}}$. Hence $r_{\alpha}$ is the spectral radius $s(f)$ of the action of $f$ on real homology. We have

THEOREM 1. For $\alpha \in \Phi_{2}, h\left(\alpha_{S^{3}}\right)=h\left(\bar{\alpha}_{S^{3}}\right)=h\left(\alpha_{S^{1}}\right)=\log r_{\alpha}$, where $r_{\alpha}$ is the spectral radius of $\alpha_{\mathbf{R}}$ or, equally, the spectral radius of the map induced by $\alpha_{S^{3}}$ (or $\alpha_{S^{1}}$ ) on real homology.

The induced action of $G l_{2} \mathbf{Z}$ on $C_{2}\left(S^{3}\right)$ preserves the function $\|x y-y x\|^{2}$. The level sets of this function define a deformation of the standard action of $G l_{2} \mathbf{Z} / \pm 1$ 
on $T^{2} / \pm 1$ to the linear action on $S^{2}$ defined by the orthogonal representation

$$
\begin{aligned}
\pm\left(\begin{array}{cc}
0 & 1 \\
-1 & 0
\end{array}\right) & \rightarrow\left(\begin{array}{ccc}
0 & 1 & 0 \\
1 & 0 & 0 \\
0 & 0 & -1
\end{array}\right) \\
\pm\left(\begin{array}{cc}
0 & 1 \\
-1 & -1
\end{array}\right) & \rightarrow\left(\begin{array}{lll}
0 & 1 & 0 \\
0 & 0 & 1 \\
1 & 0 & 0
\end{array}\right) \\
\pm\left(\begin{array}{ll}
0 & 1 \\
1 & 0
\end{array}\right) & \rightarrow\left(\begin{array}{lll}
0 & 1 & 0 \\
1 & 0 & 0 \\
0 & 0 & 1
\end{array}\right)
\end{aligned}
$$

For the last statement, one checks that $\left(\begin{array}{cc}0 & 1 \\ -1 & 0\end{array}\right)$ corresponds to $(r, s, t) \rightarrow(s, r$, $2 r s-t$ ), which has order two; hence the action of $\Phi_{2}$ factors through $G l_{2} \mathbf{Z} / \pm 1$. Then one blows up the origin, i.e. replaces it by the sphere of directions using spherical coordinates, to obtain the time $v=1$ action for our deformation. In this deformation the curvature of the quotient metric goes from a delta measure at the four vertices to a constant function.

Note that the entropy calculations of Theorem 1 are valid for $H=S O(3) \cong$ $S^{3} / \pm 1$ as well. For $H=\left(S^{3}\right)^{k}$, one has $h\left(\alpha_{H}\right)=h\left(\bar{\alpha}_{H}\right)=k \log r_{\alpha}$ and the same holds for $H$ covered by $\left(S^{3}\right)^{k}$.

To try to generalize Theorem 1 further, one might consider various unitary embeddings of the compact connected Lie group and use the resulting functions $N(x, y)=\|x y-y x\|^{2}$ to lower the dimension. This fails even for $H=S U(3)$ : since its irreducible complex representations are tensor products of the usual representation and its dual, all representations give essentially the same $N$ even though $\operatorname{dim} T=2$. One can pass to level sets of $\bar{c}$ instead. Then $S=\bar{c}^{-1}[1]$ is the image of $T \times T$, since commuting elements of $H$ lie in some conjugate of $T$, and so on this level set one has entropy $(\operatorname{dim} T) \log r_{\alpha}=h\left(\alpha_{H} \mid S\right)$. It is not clear any longer that other level sets have entropy bounded by this: They may have larger dimension than $S$, for instance, hence more expansion. Namely, if $H$ is semisimple (one easily reduces to this case), then generic level sets of $\bar{c}$ have $\operatorname{dimension} \operatorname{dim} H-\operatorname{dim} T, S$ has $\operatorname{dimension} 2 \operatorname{dim} T$ and $\operatorname{dim} H>3 \operatorname{dim} T$ unless $H$ is covered by $\left(S^{3}\right)^{k}$. We do not know the precise entropy in these cases: perhaps it is $\log r_{\alpha} \cdot \frac{1}{2}(\operatorname{dim} H-\operatorname{dim} T)$.

It would also be interesting to know whether the action of $\mathrm{PSl}_{2} \mathbf{Z}$ on each sphere $L_{v}, 0<v<1$, is ergodic.

$\mathrm{W}$. Goldman informed us that the invariance of $p(r, s, t)$ under the given action of $\mathrm{Sl}_{2} \mathbf{Z}$ was known to Fricke and Klein. Their interest lay in a certain noncompact level surface disjoint from $P$ which serves as Teichmuller space for the punctured torus. They viewed $(r, s, t)$ in terms of traces of elements in $\mathrm{Sl}_{2} \mathbf{R}$. Our application is to $S^{3}$, the compact form of the Lie algebra of $S l_{2} \mathbf{R}$, and some of the algebra carries over.

Note that for any connected compact Lie group $H$ with maximal torus $K$ and any $\alpha \in \Phi_{n}, n \geq 1$, the submanifold $K^{n} \subset H^{n}$ is invariant for $\alpha_{H}$. This gives $h\left(\alpha_{H}\right) \geq h\left(\alpha_{K}\right)=k \log r_{\alpha}$, where $k=\operatorname{dim} K$. The real cohomology ring of $H$ is a $k$-fold tensor product of cohomology rings of odd-dimensional spheres. The 
action of $\alpha_{H}$ on cohomology is the $k$-fold tensor product of the cohomology action $\alpha_{S^{1}}$ (aside from differences in grading), and so $s\left(\alpha_{H}\right)=r_{\alpha}^{k}$. Thus Shub's entropy conjecture holds for word maps. Taking $H=S O(d)$, one obtains the estimates mentioned in the introduction.

2. Isotopy classes on $S^{3} \times S^{3}$. We show that every isotopy class of $M=$ $S^{3} \times S^{3}$ contains word maps. It suffices to show this for isotopy classes that fix $H_{3}(M ; \mathbf{Z})$ (i.e., are homotopic to the identity) and word maps arising from inner automorphisms, $\alpha=i(g): a \rightarrow g a g^{-1}\left(a, g \in F_{2}\right)$. For the group $K$ of such isotopy classes, Kreck gives an exact sequence [Kr, Theorem 2, Lemma $3 \mathrm{~b}$ ]

$$
0 \rightarrow \theta_{7} \rightarrow K \stackrel{x}{\rightarrow} H^{3}(M ; \mathbf{Z}) \rightarrow 0,
$$

where $\theta_{7} \cong \mathbf{Z}_{28}$ is the group of smooth homotopy 7 -spheres. The map $\chi$ is defined as follows. Choose an $S^{3} \subset M$ with trivial normal bundle and a diffeomorphism $f$ that fixes $H_{3}(M ; \mathbf{Z})$ and leaves $S^{3}$ pointwise fixed. Then the normal part of the derivative of $f$ on $S^{3}$ defines a map $S^{3} \rightarrow G l_{3}^{+} \mathbf{R}$. Passing to the class of this map in $\pi_{3}\left(G l_{3}^{+} \mathbf{R}\right)=\pi_{3}(S O(3))=\mathbf{Z}$, we obtain a pairing $K \otimes H_{3}(M ; \mathbf{Z}) \rightarrow \mathbf{Z}$, and hence a homomorphism $\chi$.

We first compute the map $\bar{\chi}: F_{2} \rightarrow H^{3}(M ; \mathbf{Z})$ that assigns to $g \in F_{2}$ the class $\chi(f(g))$, where $f(g)=i(g)_{S^{3}}$. Choose $S^{3}=S^{3} \times 1 \subset M$. Then $f(g)$ fixes $S^{3}$ pointwise. The normal bundle of $S^{3}$ is trivialized by left translation, so that the normal differential of $f(g)$ at $(x, 1)$ corresponds to conjugation by $x^{a}$, where $a=a(g)$ is the sum of the exponents of $x_{1}$ in $g$, i.e., the $x_{1}$ component of the abelianization map $F_{2} \rightarrow \mathbf{Z}^{2}$. For $a=1$ this conjugation is the usual action of $S^{3}$ on quaternions with zero real part that gives the generator for $\pi_{3}(S O(3))$. Reasoning similarly for the other coordinate 3 -sphere $1 \times S^{3} \subset M$, we see $\bar{\chi}$ is onto with kernel the commutator subgroup $F_{2}^{\prime}=\left[F_{2}, F_{2}\right]$.

It now suffices to show that the isotopy class $\xi=[f(g)] \in K, g=c\left(x_{1}, x_{2}\right)$, corresponds to a generator of $\theta_{7}$. Note $i(g)=c\left(i\left(x_{1}\right), i\left(x_{2}\right)\right)$, so that if $d_{j}, j=1,2$, is isotopic to $f\left(x_{j}\right)$ then $d=c\left(d_{1}, d_{2}\right)$ represents $\xi$. Choose a map $u: S^{3} \rightarrow S^{3}$ homotopic to the identity so that $u^{-1}(1)$ is a neighborhood of 1 and set $d_{1}(x, y)=$ $\left(x, u(x) y u(x)^{-1}\right), d_{2}(x, y)=\left(u(y) x u(y)^{-1}, y\right)$. Note that $d_{1}$ fixes a tubular neighborhood of the coordinate sphere $1 \times S^{3}$ and preserves a tubular neighborhood of the other coordinate sphere $S^{3} \times 1$, and vice versa for $d_{2}$. Thus, $d_{1}$ and $d_{2}$ commute near $S^{3} \vee S^{3}$, so $d$ acts on $\mathbf{R}^{6}=M-S^{3} \vee S^{3}$ with compact support. Let $\phi$ be the corresponding diffeomorphism of $S^{6}=\mathbf{R}^{6} \cup\{\infty\}$. Using $\phi$ to glue two copies of the 7-disc along their boundaries gives the homotopy 7 -sphere $\delta$ corresponding to $\xi$ under Kreck's exact sequence.

Let $v \in \pi_{3} S O(3)$ be the generator mentioned above, given by the conjugation action of unit quaternions. The commutator construction above was generalized by Milnor [M, pp. 17-18] to a bilinear pairing $\pi_{3} S O(3) \otimes \pi_{3} S O(3) \rightarrow \theta_{7}$, so (up to sign) $v \otimes v$ goes to $\delta$. Milnor notes that $\delta$ generates $\theta_{7}$, and this can be checked by showing that the Eells-Kuiper homomorphism $\mu: \theta_{7} \rightarrow \mathbf{Q} / \mathbf{Z}$ takes the value $\pm 1 / 28$ on $\delta$; cf. $[\mathbf{L}]$.

Let $\pi=\pi_{0} \operatorname{Diff} M$. Let $W: \Phi_{2} \rightarrow \pi$ be the homomorphism sending $\alpha \in \Phi_{2}$ to the isotopy class of the word map $\alpha_{S^{3}}$. We have shown $W$ is onto and $\operatorname{Ker} W \subset F_{2}^{\prime}$ with $F_{2}^{\prime} / \operatorname{Ker} W \cong \mathbf{Z}_{28}$. We now compute this kernel $S=\operatorname{Ker} W$. 
Let $c_{i j}=c\left(x_{1}^{i}, x_{2}^{j}\right) \in F_{2}^{\prime}$. Then using the bilinearity of Milnor's pairing, one sees that $W\left(c_{i j}\right)=i j \delta \in \theta_{7} \subset \pi$. Now identify $F_{2}$ with $\pi_{1} X, X$ a figure eight, so that $F_{2}^{\prime}=\pi_{1} \tilde{X}$ where the grid $\tilde{X}=\mathbf{R} \times \mathbf{Z} \cup \mathbf{Z} \times \mathbf{R} \subset \mathbf{R}^{2}$ is the maximal abelian covering of $X$. Then $W: F_{2}^{\prime} \rightarrow \theta_{7}$ induces $\bar{W}: H_{1}(\tilde{X} ; \mathbf{Z}) \rightarrow \mathbf{Z}_{28}$. The class $c_{i j}, i, j \in \mathbf{Z}-\{0\}$, corresponds to a rectangular loop in $\tilde{X}$ based at $(0,0)$. These loops clearly generate $H_{1} \tilde{X}$. Thus $\bar{W}$ is the homomorphism which assigns to a loop $\gamma$ its enclosed area $A(\gamma)$ reduced modulo 28 . Note that $\operatorname{ker} A=\left[F_{2}, F_{2}^{\prime}\right]$ and that $F_{2} /\left[F_{2}, F_{2}^{\prime}\right]$ is the nilpotent group

$$
H_{\mathbf{Z}}=\langle x, y, z \mid z=c(y, z), c(x, z)=1=c(y)\rangle .
$$

Clearly $H_{\mathbf{Z}}$ is isomorphic to the integral Heisenberg group, i.e., the integral $3 \times 3$ matrices of form $I+N$ with $N$ strictly upper triangular. We have shown

THEOREM 2. There is an exact sequence

$$
1 \rightarrow S \rightarrow \Phi_{2} \stackrel{W}{\rightarrow} \pi_{0} \operatorname{Diff}\left(S^{3} \times S^{3}\right) \rightarrow 1,
$$

where $\left[F_{2}, F_{2}^{\prime}\right] \subset S \subset F_{2}^{\prime} \subset F_{2} \subset \Phi_{2}$. In the quotient $F_{2}^{\prime} /\left[F_{2}, F_{2}^{\prime}\right] \cong \mathbf{Z}, S /\left[F_{2}, F_{2}^{\prime}\right]$ corresponds to the subgroup $28 \mathrm{Z}$.

In particular, $K=H_{\mathbf{z}} / z^{28}$ is nilpotent and $\chi$ is a central extension. These properties of $K$ (and their analogues for other manifolds of high connectivity) are due to Bernhard Schmidt [Sc].

Let $J=\left(\begin{array}{cc}0 & -1 \\ 1 & 1\end{array}\right) \in G l_{2} \mathbf{Z}, J^{6}=I$. Then we show for the natural map $\Phi_{2} \rightarrow G l_{2} \mathbf{Z}$ induced by $F_{2} \rightarrow \mathbf{Z}^{2}$ :

LEMMA. If $B \in \Phi_{2}$ covers $J$, then $B^{6} \notin S$.

The next corollary follows immediately from the lemma.

COROLlaRY. The natural map $\pi \rightarrow G_{2} \mathbf{Z}$ from isotopy classes to homotopy classes does not split.

Let $A \in \Phi_{2}$ with $A x=y, A y=y x^{-1}$. Then $A$ covers $J$ and $A^{3}=i\left(y x^{-1}\right) \circ \phi$, where $\phi x=x^{-1}, \phi y=y^{-1}$. By Nielsen's result any $B$ covering $J$ is of the form $B=i(g) \circ A$ for some $g \in F_{2}$. Then $B^{3}=i(h) \circ \phi$ for $h=g \cdot A g \cdot A^{2} g \cdot y x^{-1}$.

Since $I+J+J^{2}$ has even entries, $h \equiv x^{i} y^{j} z^{k} \bmod S$ with $i, j$ odd. Thus

$$
B^{6}=\left(B^{3}\right)^{2}=i(h \cdot \phi(h)) \circ \phi^{2}=i\left(x^{i} y^{j} z^{k} x^{-i} y^{-j} z^{k}\right) \equiv i\left(z^{l}\right) \bmod S,
$$

for $l$ odd. Since $z$ has even order $\bmod S$ (namely $\left.28=\left|\theta_{7}\right|\right), B^{6} \notin S$, as desired. This proves the lemma and corollary.

Note the essential use of the nontriviality of the subgroup $\theta_{7}$ of homotopy 7 spheres. Indeed for the quotient group $\pi / \theta_{7}$, the natural map $\pi / \theta_{7} \rightarrow G l_{2} \mathbf{Z}$ does split, meaning that the usual action of $G l_{2} \mathbf{Z}$ on $\mathbf{Z}^{2}$ lifts to an action by automorphisms of $H_{\mathbf{z}}$. This can easily be seen by noting that the real Heisenberg group is the free two-step nilpotent Lie group generated by $\mathbf{R}^{2}$ and that $H_{\mathbf{Z}}$ is generated by $\mathbf{Z}^{2} \subset \mathbf{R}^{2}$.

Also note that $B^{6}$ has infinite order in $\Phi_{2}$ (since $F_{2}$ is torsionfree and centerless), so the word map $B_{S^{3}}$ has infinite order for all $B$ (the only trivial word map on $S^{3}$ comes from $1 \in \Phi_{2}$ ). We do not know if there is a periodic map of $M$ inducing $J$ on $H_{3} M$; if it is smooth, then we see its period must be divisible by 24 . 
3. Isentropic approximation. By the results of $\S 1$, we see that every isotopy class on $M$ contains diffeomorphisms of entropy $\log s$, where $s$ is the spectral radius of the action on real homology. Thus if Shub's entropy conjecture holds on $M$, these word maps minimize entropy in their isotopy class. But it is not even known whether the entropy function on Diff $M$ is lower semicontinuous at a word map, aside from the trivial case of zero entropy.

We now show that a word map on two letters for $S^{3}$ or $S O(3)$ can be isotoped to an isentropic (equal entropy) fitted diffeomorphism. It suffices to work on $H=$ $S O(3)$, because the isotopy can be lifted to $M$.

The function

$$
(\operatorname{Re} x)^{2}+(\operatorname{Re} y)^{2}+(\operatorname{Re} x y)^{2}-2(\operatorname{Re} x)(\operatorname{Re} y)(\operatorname{Re} x y)
$$

on $M$ that (essentially) arose in $\S 1$ induces a function $g: H^{2} \rightarrow[0,1]$ regular over $(0,1)$. The critical level $\tilde{L}_{0}=g^{-1} 0$ is a submanifold consisting of a single $H$ orbit corresponding to pairs of $180^{\circ}$ rotations about perpendicular axes. The other critical level $\tilde{L}_{1}=g^{-1} 1$ corresponds to pairs of commuting rotations. $\tilde{L}_{1}-(I, I)$ is a smooth submanifold. By assigning to a commuting pair $\neq(I, I)$ the common axis of rotation, one obtains a flat fibration of $\tilde{L}_{1}-(I, I)$ over $\mathbf{R} P^{2}$, with fiber a punctured torus $T^{2}-0$ and monodromy \pm 1 . There is a vector field $X$ on $H^{2}$ such that

(a) $X$ is $H$-invariant.

(b) $X g \leq 0$ with $X g=0$ precisely on $\tilde{L}_{0} \cup \tilde{L}_{1}$.

(c) $X=0$ on $\tilde{L}_{0}$ and on those points of $\tilde{L}_{1}$ outside an $\varepsilon$-neighborhood of $(I, I)$.

(d) $(I, I)$ is a source of $X$.

(e) $\tilde{L}_{1}-(I, I)$ is a normally hyperbolic repeller, and $\tilde{L}_{0}$ is a normally hyperbolic attractor.

Let $\phi_{t}$ be the corresponding flow. For $t \gg r_{\alpha}>1, \beta=\phi_{t} \circ \alpha_{H}$ has a chain recurrent set $R(\beta)$ consisting of $\tilde{L}_{0},(I, I)$ and a family $M_{1} \subset \tilde{L}_{1}-(I, I)$ of $D A$ attractors indexed by $\mathbf{R} P^{2}$. Moreover, $\beta\left|\tilde{L}_{0}=\alpha_{H}\right| L \tilde{L} 0$ has finite order. Choose an equivariant Morse function on $\tilde{L}_{0}$ and extend its gradient vector field to a vector field $Y_{0}$ supported near $\tilde{L}_{0}$. Then choose a Morse function on $\mathbf{R} P^{2}$, lift its gradient vector field to $M_{1}$ using the flat connection and extend to a vector field $Y_{1}$ supported on a neighborhood of $M_{1}$. Let $\psi_{t}$ be the flow generated by $Y_{0}+Y_{1}$. Then for $\varepsilon>0$ and small enough, $\gamma=\psi_{\varepsilon} \circ \beta$ is Axiom A-No Cycles, with $R(\gamma)$ consisting of the source $(I, I)$, finitely many points in $\tilde{L}_{0}$ and finitely many $D A$ attractors in $M_{1}$. Finally, double $D A$ these $D A$ attractors to get an Axiom A-No Cycles map $\delta$ with $R(\delta)$ zero dimensional. The Artin-Mazur zeta functions satisfy

$$
\varsigma(\delta)=\varsigma\left(\alpha_{S^{1}}\right)^{k} \cdot R
$$

where $k$ is the number of critical points of the Morse function on $\mathbf{R} P^{2}$ and $R$ is a finite product of terms $\left(1-t^{n}\right)^{ \pm 1}$, so $h(\delta)=h\left(\alpha_{S^{1}}\right)=\log r_{\alpha}$. It is then easy to find a fitted diffeomorphism $\Omega$-conjugate to $\delta$ and isotopic to $\delta$.

The case $r_{\alpha}=1$ requires an isotopy to Morse-Smale. The same procedure clearly works: no $D A$ attractors arise, and one has to use instead a gradient flow on $T^{2}$. 
We have shown

THEOREM 3. Every isotopy class of $S^{3} \times S^{3}$ has a fitted representative $f$ with entropy $\log s(f)$. The same holds for any isotopy class of $S O(3) \times S O(3)$ that contains a word map.

See $[$ F1] for results and a discussion of the corresponding question for word maps on $S^{1}$, i.e., toral automorphisms. Even for $T^{3}$ some isotopy classes are not known to have a fitted representative with entropy $\log s$. Specifically for $\operatorname{det}(x-A)=$ $x^{3}-x-1, A \in S l(3, \mathbf{Z})$, it is not known whether the linear map $A$ has an isentropic fitting. On $T^{4}$ there are examples where no isentropic Axiom A representative exists in the homotopy class of a certain linear map [F2].

\section{REFERENCES}

[B] R. Bowen, Entropy for group endomorphisms and homogeneous spaces, Trans. Amer. Math. Soc. 153 (1971), 401-414.

[F1] D. Fried, Isentropic fitting of Anosov automorphisms, Ergodic Theory Dynamical Systems 2 (1982), 173-183.

[F2] _ Efficiency vs. hyperbolicity on tori, Lecture Notes in Math., vol. 819, Springer, 1980, pp. 179-189.

[K] S. Katok, The estimation from above for the topological entropy of a diffeomorphism, Lecture Notes in Math., vol. 819, Springer, 1980, pp. 258-264.

[Kr] M. Kreck, Isotopy classes of diffeomorphisms of $(k-1)$-connected almost-parallelizable $2 k$-manifolds, Lecture Notes in Math., vol. 763, Springer, 1979, pp. 643-663.

[L] J. Levine, Inertia groups of manifolds and diffeomorphisms of spheres, Amer. J. Math. 92 (1970), 243-258.

[MKS] W. Magnus, A. Karrass and D. Solitar, Combinatorial group theory, Dover, New York, 1976.

[M] J. Milnor, Differential structures, Princeton Univ., mimeographed notes, 1961.

[N] J. Nielsen, Isomorphismen der allgemeinen unendlichen Gruppe mit zwei erzeugenden, Math. Ann. 78 (1918), 385-397.

[Sc] B. Schmidt, Diffeomorphismen auf hochzusammenhängen Mannigfaltigkeiten, Diplomarbeit, Johannes Gutenberg-Universität Mainz, 1982.

[S] M. Shub, Dynamical systems, filtrations and entropy, Bull. Amer. Math. Soc. 80 (1974), 27-41.

DePaRtMENT OF MATHEMATICS, Boston UNIVERSity, 111 CUMmington Street, BOSTON, MASSACHUSETTS 02215 